\title{
RELCASI
}

Volume 2 | Issue 1

Article 4

2009

\section{Distribución Geográfica de los Estudiantes de Ingeniería del ITAM en la Ciudad de México: Caso de Estudio}

\author{
Marcelo Mejia \\ Instituto Tecnologico Autonomo de Mexico, marcelo@itam.mx \\ Quauhtli Martínez \\ Instituto Tecnológico Autónomo de México, quauhtlimtz@gmail.com
}

Follow this and additional works at: https://aisel.aisnet.org/relcasi

\section{Recommended Citation}

Mejia, Marcelo and Martínez, Quauhtli (2009) "Distribución Geográfica de los Estudiantes de Ingeniería del ITAM en la Ciudad de México: Caso de Estudio," RELCASI: Vol. 2 : Iss. 1 , Paper 3.

DOI: $10.17705 / 1$ relc.00008

Available at: https://aisel.aisnet.org/relcasi/vol2/iss1/4

This material is brought to you by the AIS Journals at AIS Electronic Library (AISeL). It has been accepted for inclusion in RELCASI by an authorized administrator of AIS Electronic Library (AISeL). For more information, please contact elibrary@aisnet.org. 


\section{R E L C A S I $\quad 02 \quad 01 \quad 2009$}

\section{REVISIA HAINOAMERICANA}

Y DEL CARIBE DE LA

\section{ASOCIACIÓN DE SISTEMAS

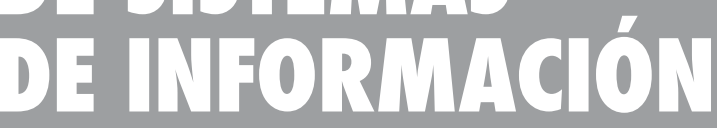

11 - Participación de los Usuarios en la Evaluación de la Calidad de los Sistemas de Información Juan Manuel Gómez Reynoso y Mónica Brizuela Sandoval

35- Impacto del Adiestramiento, Habilidades en Tecnología de la Información y Gerencia de Proyectos en el Éxito de Implementaciones de Sistemas Integrados ERP Miguel Maldonado y Martin Santana

$55 \cdot$ Distribución Geográfica de los Estudiantes de Ingeniería del ITAM en la Ciudad de México:

Caso de Estudio Marcelo Mejia y Quauhti Martinez 


\section{$\begin{array}{llllllllll}R & E & L & C & A & S & \mid & 02 & 01 & 2009\end{array}$}

\section{REVISTA LATLNOAMERICANA}

ASOCAACÓ Y DE CARTB DE LA DE SISIEMAS DE INFORMAción

Editor:

Carlos Ferran 
Editor:

Carlos Ferran

Penn State Great Valley Pennsylvania State University 30 E Swedesford Road Malvern, PA 19355 U.S.A. cferran@psu.edu

Comité Editorial:

Carlos Dorantes Tecnológico de Monterrey, México cdorante@itesm.mx

Carlos Ferran Pennsylvania State University,

USA cferran@psu.edu

Martha Garcia-Murillo Syracuse University, USA mgarciam@syr.edu

David Montesinos INCAE, Costa Rica

David.Montesinos@incae.edu

Carlos J. Navarrete California State Polytechnic University, USA cjnavarrete@csupomona.edu

James B. Pick University of Redlands, USA James_Pick@redlands.edu

Guillermo Rodríguez-Abitia Universidad Nacional Autonoma de México grdrz@unam.mx

Volumen 2 Número 1, 2009 Portada: Maria Elena Repiso C) 2009 RELCASI ISSN 1937-8823

(en línea) ISSN 1937-8831 www.relcasi.org

\section{Revista Latinoamericana} y del Caribe de la Asociación de Sistemas de Información RELCASI

\section{TABLA DE CONTENIDO}

Volumen 2 Número 1, 2009

NOTA EDITORIAL

PARTICIPACIÓN DE LOS USUARIOS EN LA EVALUACIÓN DE LA CALIDAD DE LOS SISTEMAS DE INFORMACIÓN

Juan Manuel Gómez Reynoso y

Mónica Brizuela Sandoval

IMPACTO DEL ADIESTRAMIENTO, HABILIDADES EN TECNOLOGÍA DE LA INFORMACIÓN Y GERENCIA DE PROYECTOS EN EL ÉXITO DE IMPLEMENTACIONES DE SISTEMAS INTEGRADOS ERP

Miguel Maldonado y

Martín Santana

DISTRIBUCIÓN GEOGRÁFICA DE LOS ESTUDIANTES DE INGENIERIA DEL ITAM EN LA CIUDAD DE MÉXICO: CASO DE ESTUDIO

Marcelo Mejía y

Quauhtli Martinez

POLÍTICA EDITORIAL 
Editor-in-Chief:

Carlos Ferran

Penn State Great Valley Pennsylvania State University 30 E Swedesford Road Malvern, PA 19355 U.S.A. cferran@psu.edu

Editorial Board:

Carlos Dorantes Tecnológico de Monterrey, México cdorante@itesm.mx

Carlos Ferran Pennsylvania State University, USA cferran@psu.edu

Martha Garcia-Murillo Syracuse University, USA mgarciam@syr.edu

David Montesinos INCAE, Costa Rica David.Montesinos@incae.edu

Carlos J. Navarrete California State Polytechnic University, USA cjnavarrete@csupomona.edu

James B. Pick University of Redlands, USA James Pick@redlands.edu

Guillermo Rodríguez-Abitia Universidad Nacional Autonoma de México grdrz@unam.mx

Volume 2 Number 1, 2009 (C) 2009 RELCASI Cover: Maria Elena Repiso ISSN 1937-8823 (on-line) ISSN 1937-8831 www.relcasi.org

\section{Revista Latinoamericana y del Caribe de la Asociación de Sistemas de Información RELCASI}

TABLE OF CONTENT

Volume 2 Number 1, 2009

EDITORIAL

END-USER PARTICIPATION IN INFORMATION SYSTEMS QUALITYEVALUATION

Juan Manuel Gómez Reynoso y

Mónica Brizuela Sandoval

THE IMPACT OF TRAINING, IT SKILLS AND PROJECT MANAGEMENT ON ERP SYSTEMS IMPLEMENTATIONS SUCCESS

Miguel Maldonado y

Martín Santana

GEOGRAPHICAL DISTRIBUTION OF ITAM'S ENGINEERING STUDENTS IN MEXICO CITY: A CASE STUDY

55

Marcelo Mejía y

Quauhtli Martínez

EDITORIAL POLICY

74

CALL FOR ARTICLES 


\title{
DISTRIBUCIÓN GEOGRÁFICA DE LOS ESTUDIANTES DE INGENIERÍA DEL ITAM EN LA CIUDAD DE MÉXICO: CASO DE ESTUDIO
}

\section{Geographical Distribution of ITAM's Engineering Students in Mexico City: A Case Study}

\author{
Marcelo Mejía \\ Instituto Tecnológico Autónomo de México, marcelo@itam.mx
}

\section{Quauhtli Martínez}

Instituto Tecnológico Autónomo de México, quauhtlimtz@gmail.com

\section{RESUMEN}

El presente caso de estudio analiza la distribución geográfica de los estudiantes de Ingeniería del ITAM en la Ciudad de México. Basándose en la visualización de la ubicación de los alumnos actuales y en la distribución de la población y de la riqueza en las delegaciones del Distrito Federal el estudio sugiere que la distancia de los hogares de los candidatos potenciales a ingresar y su nivel socio económico son dos factores importantes en la decisión de inscribirse al Instituto.

Palabras Claves: Distribución espacial, GIS, estudiantes de Ingeniería, Ciudad de México.

\begin{abstract}
This case study analyzes the geographic distribution of ITAM's engineering students in Mexico City. Based on the visualization of the location of our current students and the population and wealth distribution in the boroughs of the Mexican Federal District, the study suggests that the distance from the homes of potential applicants and their socio-economic level are two important factors in the decision of candidates to enroll in our institution.
\end{abstract}

Keywords: Spatial Distribution, GIS, Engineering students, Mexico City.

\section{INTRODUCCIÓN}

El número de alumnos interesados en estudiar una licenciatura relacionada con Tecnologías de Información ha declinado fuertemente en los últimos años. Entre 2002 y 2005 se estima que la demanda por estudiar Sistemas de Información en Estados Unidos cayó aproximadamente 50\% (George, 
Valacich \& Valor, 2005) y que entre 2001 y 2007 la disminución pudo haber alcanzado entre 70 y $80 \%$ en algunas partes del mundo (Granger, et al., 2007a). De igual manera, los alumnos interesados en estudiar Ciencias de la Computación en universidades de Estados Unidos disminuyeron 70\% entre 2000 y 2005 y la caída continuó en 2006 y 2007 (Vegso, 2008). Para revertir esta tendencia se han realizado muchos esfuerzos, entre los que destacan la creación del wiki "The Enrollment Crisis in Computing" (http://enrollments.aisnet.org/) y las propuestas por utilizar a los mejores profesores en los cursos introductorios de Sistemas de Información (Looney y Akbulut, 2007), incrementar la visibilidad del área de estudio mediante campañas de mercadotecnia y promoción (Granger, et al., 2007b), cambiar el lenguaje con el que se enseña a programar (http://www.alice.org/) e introducir a los niños a las Ciencias de la Computación sin utilizar una computadora (http://csunplugged.com).

En el área metropolitana de la Ciudad de México, esta crisis de inscripciones se combina con un aumento considerable en el número de planteles universitarios que se han abierto en los últimos 10 años para crear un entorno de competencia fuerte por reclutar alumnos. Dada la población y la extensión geográfica de la Ciudad de México, cuatro instituciones de educación superior públicas y algunas privadas cuentan con varios planteles (entre 2 y 10) distribuidos en el área metropolitana. La creación de nuevos planteles y programas de estudio obedeció al crecimiento de la demanda por educación superior (ANUIES, 2004) pero nunca contempló la disminución que se tendría en el número de inscripciones a programas en el área de Tecnologías de Información.

El desinterés creciente de los alumnos del nivel bachillerato por estudiar una carrera de Ingeniería relacionada con Tecnologías de Información, el aumento considerable en el número de universidades que ofrecen estudios de nivel licenciatura en la Ciudad de México y el bajo crecimiento económico del país han creado un nivel de competencia alto por atraer estudiantes talentosos a las universidades privadas (que en México atienden a poco más del $30 \%$ de los alumnos).

En este contexto, el Instituto Tecnológico Autónomo de México (ITAM) ha realizado diversos proyectos para tratar de enfocar sus esfuerzos de reclutamiento. El presente trabajo se enmarca dentro de estos esfuerzos y utilizando un Sistema de Información Geográfica (SIG) complementa un estudio anterior (Barrera, 2000) que identificó, por medio de técnicas de inteligencia artificial, las escuelas preparatorias (bachilleratos) de las cuales provienen los alumnos de mayor potencial para las carreras de Ingeniería. Consideramos que el uso de herramientas de SIG podrán ser un elemento que universidades en otras ciudades metropolitanas de América Latina pueden utilizar para determinar si este es un factor de relevancia en el reclutamiento de estudiantes. 
En la siguiente sección del artículo se revisa la literatura relevante sobre el uso de SIGs en el estudio de la distribución geográfica de alumnos. Posteriormente se presentan los objetivos del trabajo, se describe la metodología utilizada en la investigación y se reportan los principales resultados obtenidos. Finalmente, se presentan algunas conclusiones del estudio.

\section{REVISIÓN DE LA LITERATURA}

Los SIG se han utilizado con éxito en una gran variedad de áreas dentro de las empresas (Pick, 2005) y también en aplicaciones relacionadas con la inscripción de estudiantes a escuelas en los Estados Unidos. Uno de los primeros trabajos en este campo relacionó un sistema de información que contenía características de los estudiantes y sus direcciones con una base de datos geográfica para hacer proyecciones de corto plazo en el número de inscripciones por grado y grupo étnico en áreas geográficas pequeñas en la ciudad de Iowa (Rushton, Armstrong \& Lolonis, 1995).

En otro trabajo pionero, investigadores de la Universidad Estatal de Ohio (Marble, Mora \& Herries, 1995) lanzaron en 1995 un estudio para explorar la factibilidad del uso de tecnología SIG en su proceso de admisión mediante la codificación geográfica de datos provenientes de las 80,000 solicitudes de información y de admisión que procesaron en los dos años anteriores. Dos años después, elaboraron un modelo conceptual del proceso de admisión, identificaron áreas de oportunidad para los SIG y analizaron la estructura demográfica y geográfica de los candidatos (Herries y Marble, 1997). Como resultado de estos esfuerzos, identificaron los perfiles geodemográficos de los candidatos que con mayor probabilidad se inscribirían a la universidad (Marble, Mora \& Granados, 1997) y fueron capaces de identificar en qué zonas de Ohio sus esfuerzos de reclutamiento estaban siendo eficaces y en cuáles no.

Por su parte, un estudio cartográfico de las áreas de mercado de las 14 universidades del sistema estatal de educación superior de Pennsylvania mostró que cada universidad domina su área local (Martin, 2000). Investigadores de la Universidad Estatal de Bowling Green constataron también el impacto que tiene la distancia a la universidad en el número de estudiantes inscritos y analizaron qué condados del estado de Ohio proveen a su universidad de más (o menos) estudiantes que los esperados, tomando en cuenta la población que tienen y la distancia a la que se encuentran (Zhou y Wu, 2000). La Universidad de Kutztown en Pennsylvania utilizó un SIG y una definición de demanda potencial por distrito escolar (directamente proporcional al número de estudiantes de preparatoria en el distrito e inversamente proporcional a la distancia entre el distrito y la universidad) para identificar en qué áreas debía enfocar sus esfuerzos de reclutamiento de minorías (Martin, 2001) e incorporó en el 
cálculo del número esperado de estudiantes el tiempo de transporte a la universidad en lugar de la distancia en línea recta (Martin, 2002).

De la misma manera, el campus de Pomona de la Universidad de DeVry (Price, 2004) utilizó un SIG para analizar los datos históricos de sus estudiantes y datos públicos del sistema educativo de California para identificar, dentro de un radio geográfico determinado, las preparatorias que serían el objetivo de sus esfuerzos de reclutamiento para los programas de Ingeniería y Tecnología. La Universidad Estatal de California en Dominguez Hills utiliza datos sobre la matrícula de preparatorias y colegios comunitarios, y las tendencias recientes en solicitudes a campus cercanos para determinar las características geodemográficas de posibles candidatos futuros que guíen iniciativas de reclutamiento y desarrollo de programas académicos (Christie y Ferris, 2004).

Las ideas presentadas en los estudios relacionados con el uso de SIGs en universidades de Estados Unidos pueden combinarse y adaptarse al entorno de una universidad privada en la Ciudad de México. Para propósitos de reclutamiento, es importante visualizar la distribución geográfica actual de los alumnos del ITAM y saber si los resultados de dominio de localidad encontrados en universidades de Estados Unidos se aplican en el contexto de un área metropolitana grande de Latinoamérica. En particular, resulta interesante estudiar el impacto que tiene la distancia al ITAM en el número de estudiantes inscritos y analizar qué delegaciones políticas del Distrito Federal proveen al ITAM de más (o menos) estudiantes que los esperados, tomando en cuenta la población que tienen y la distancia a la que se encuentran. Como aportación original, se debe analizar cómo influye el nivel socioeconómico de los hogares de los estudiantes en los resultados del estudio.

Los resultados y conclusiones obtenidos en el presente artículo podrían adaptarse y aplicarse a otros casos particulares en universidades localizadas en áreas metropolitanas grandes de Latinoamérica como São Paulo, Buenos Aires, Río de Janeiro, Bogotá, Lima, Santiago o Caracas, en los que también exista una competencia fuerte por captar más y mejores alumnos.

\section{OBJETIVO}

El objetivo del presente artículo es estudiar la distribución geográfica de los alumnos inscritos actualmente en un programa de Ingeniería en el ITAM con el propósito de analizar nuestro mercado y poder enfocar mejor los esfuerzos locales de reclutamiento necesarios para incrementar el número de alumnos que solicitan inscripción al Instituto.

Sabiendo que los estudiantes del ITAM que son originarios de la Ciudad de México viven en casa de sus padres mientras cursan sus estudios de 
Ingeniería, la pregunta de investigación que se quiere responder es: ¿qué tan importantes son la distancia a la que se encuentra del ITAM el hogar de un alumno de preparatoria y el nivel socioeconómico de su familia en su decisión de inscripción a alguno de los programas de Ingeniería que ofrece el Instituto?

Se analizan específicamente las siguientes interrogantes: ¿en qué parte de la Ciudad de México se encuentran concentrados los alumnos actuales? ¿Cómo afecta en el número de alumnos esperados de cada una de las 16 delegaciones políticas en que se encuentra dividido el Distrito Federal la distancia a la que se encuentra, su población total, o su población en edad universitaria? ¿Existe alguna relación entre el porcentaje de alumnos de Ingeniería en el ITAM pertenecientes a cada delegación con su cercanía y/o nivel socioeconómico?

El estudio de la distribución geográfica es importante para saber qué partes de la ciudad proporcionan pocos estudiantes y por lo tanto en dónde se podría implementar un programa de reclutamiento adicional. El caso de estudio se enfoca en las variables distancia y nivel socioeconómico ya que el ITAM es una institución de educación superior privada que compite por los mejores alumnos con un gran número de universidades públicas y privadas presentes en el área metropolitana de la Ciudad de México. La distancia de los hogares de los alumnos al ITAM es una variable importante en el estudio ya que, debido a las graves deficiencias del transporte público y a los problemas cotidianos de exceso de tráfico, un alumno podría preferir estudiar en otra universidad tomando en cuenta sólo el factor de cercanía.

\section{METODOLOGÍA}

En base al objetivo del trabajo y a la revisión de la literatura especializada, se definieron los datos que deberían reunirse para realizar el estudio. Estos datos se obtuvieron de diferentes fuentes. Primeramente se realizó una encuesta vía Web a los alumnos de Ingeniería del ITAM para obtener las coordenadas geográficas exactas de su casa y de su preparatoria de proveniencia. La encuesta se aplicó en una prueba piloto a un grupo reducido de alumnos y a los Directores de los programas de Ingeniería para mejorar la información de la página y su usabilidad. Los alumnos utilizaron Google Earth para obtener las coordenadas pedidas. De los 96 alumnos que contestaron la encuesta, sólo 43 proporcionaron datos correctos dentro de los campos de latitud y longitud, los demás registros se consideraron inválidos debido a que la latitud y la longitud eran iguales a los límites permitidos por la página. Este número representa el 10\% de la población total. Para obtener más datos, se obtuvo del sistema de administración escolar el código postal de los hogares de todos los alumnos de Ingeniería y de su preparatoria de proveniencia. Estos datos de 
localización son menos precisos que los obtenidos mediante la encuesta pero incluyen al universo completo de la población estudiada.

Por otro lado, se obtuvo información estadística del Mapa Mercadológico de la Ciudad de México (2004) y del Sistema Municipal de Base de Datos del Instituto Nacional de Estadística, Geografía e Historia (INEGI, 2007). La información obtenida para cada delegación política del Distrito Federal incluye la población total, la población entre 18 y 25 años, la población que estudia Ingeniería y la población en cada uno de los niveles socioeconómicos definidos por la Asociación Mexicana de Agencias de Investigación de Mercados y Opinión Pública (AMAI, http://www.amai.org/).

El índice de nivel socioeconómico utilizado clasifica a los hogares en México en seis niveles a partir de un árbol de asignaciones que considera diferentes variables como la escolaridad del jefe del hogar, el número de habitaciones, el número de baños con regadera, el tipo de piso, el número de focos, o la posesión de auto, lavadora de ropa automática y computadora personal, entre otros productos. En el 2005, el ingreso familiar mensual por nivel socioeconómico en el país se estimaba de $\$ 0.00$ a $\$ 2,699$ para el nivel E; de $\$ 2,700$ a $\$ 6,790$ para el nivel $D$; de $\$ 6,800$ a $\$ 11,599$ para el nivel $\mathrm{D}+$; de $\$ 11,600$ a 34,999 para el nivel $\mathrm{C}$; de $\$ 35,000$ a $\$ 84,999$ para el nivel $\mathrm{C}+\mathrm{y}$ de $\$ 85,000$ o más para el nivel A/B. ${ }^{1}$. Estos datos son importantes en este caso de estudio ya que pueden compararse con el costo (promedio) de un semestre de un programa de Ingeniería en el ITAM que es de $\$ 50,000$.

A partir de los datos recolectados se procedió a visualizar la información por medio de mapas (en MapPoint de Microsoft), tablas y gráficas (en Excel). Esta visualización permitió encontrar patrones sencillos en la información y analizarlos para contestar las interrogantes de investigación.

\section{RESULTADOS Y DISCUSIÓN}

Como se muestra en la Figura 1, el ITAM se encuentra en el sur poniente de la mancha urbana del área metropolitana de la Ciudad de México, constituida por las 16 delegaciones políticas del Distrito Federal, 40 municipios del Estado de México y 1 municipio del Estado de Hidalgo (Green y Pick, 2006). La mayor parte de los alumnos de Ingeniería se distribuyen en el Distrito Federal como se observa en la Figura 2. Algunas de las zonas "vacías" en el mapa pueden explicarse por la presencia del Aeropuerto Internacional Benito Juárez, de la Ciudad Universitaria, de grandes parques, y de zonas industriales donde no hay asentamientos de población. Sin embargo, en el resto del mapa existen zonas donde existe

${ }^{1}$ El tipo de cambio se ha mantenido relativamente estable en los últimos años en aproximadamente $\$ 10.9$ por dólar. 
mayor densidad de población de alumnos que en otras. Para estudiar esta diferencia de densidad, se analizan los datos con los que contamos.

Primeramente, se estudian los datos provenientes de la encuesta Web aplicada. Se trata entonces de una muestra de la población con datos exactos de distancia. La gráfica de barras de la Figura 3 muestra que mientras mayor es la distancia entre los hogares de los alumnos y el ITAM, menor es el número de alumnos inscritos en los programas de Ingeniería. Con el propósito de saber si los alumnos que vivieron cerca de su preparatoria decidieron entrar al ITAM dado que también estaba cerca de su lugar de residencia se buscó una relación significativa entre las distancias de los hogares de los alumnos al ITAM y a sus preparatorias de procedencia mediante una gráfica de dispersión, pero no se encontró.

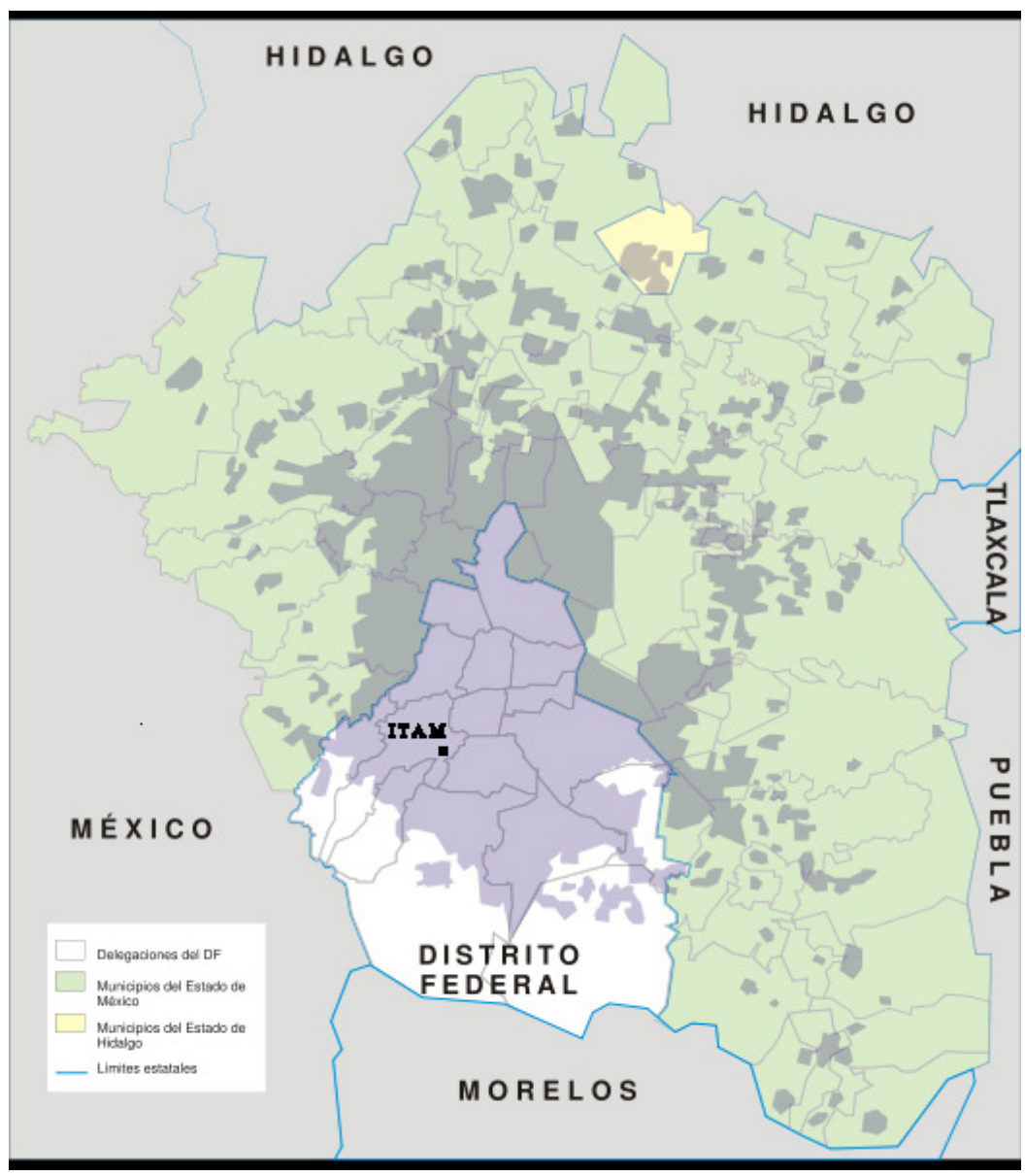

Figura 1. Localización del ITAM dentro del área metropolitana de la Ciudad de México 


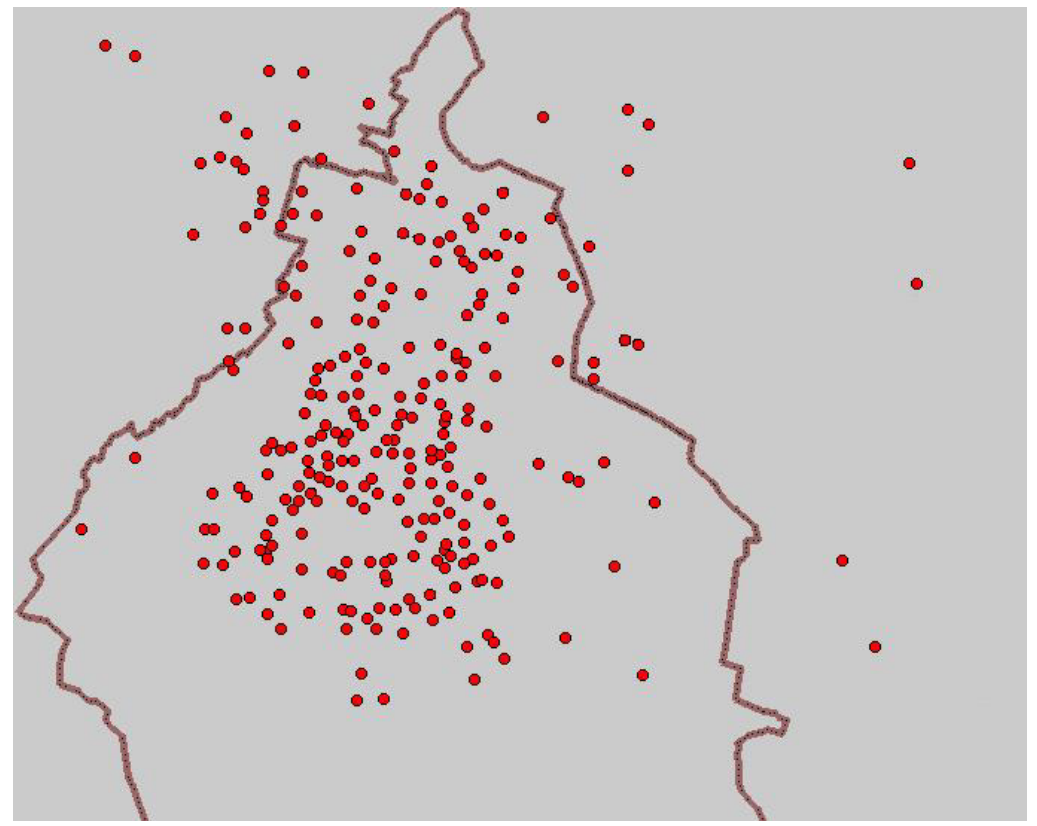

Figura 2. Localización de los estudiantes actuales de Ingeniería

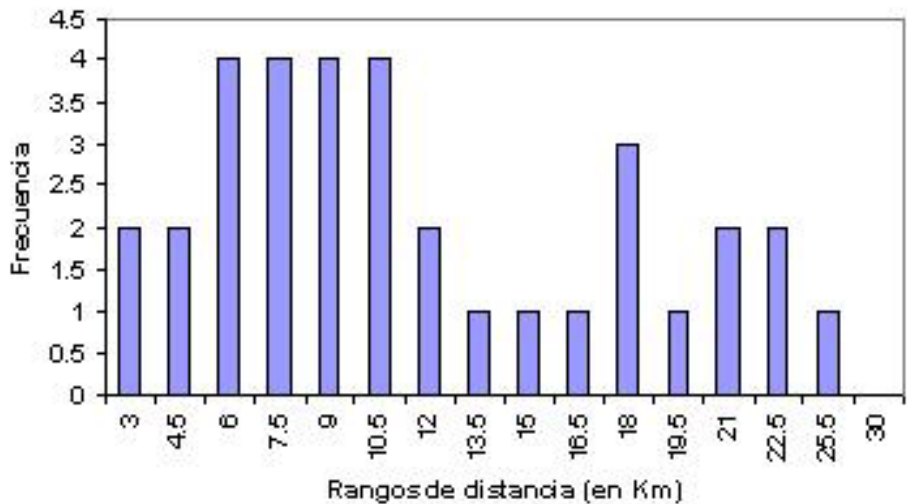

Figura 3. Número de alumnos inscritos en función de la distancia (en km) de los hogares al ITAM

Para continuar con el análisis del efecto que tiene la distancia al ITAM en el número de alumnos inscritos se utilizan los datos disponibles a nivel delegación política del Distrito Federal y los datos de ubicación de todos los alumnos de Ingeniería que viven en estas delegaciones. 


\begin{tabular}{|c|c|c|c|c|c|c|}
\hline Delegación & $\begin{array}{l}\text { Número de } \\
\text { alumnos }\end{array}$ & $\begin{array}{c}\text { Porcentaje de } \\
\text { alumnos }\end{array}$ & $\begin{array}{c}\text { Población } \\
\text { total }\end{array}$ & $\begin{array}{c}\text { Porcentaje de la } \\
\text { población }\end{array}$ & $\begin{array}{c}\text { Población total } \\
\text { entre } 15 \text { y } 24 \text { años }\end{array}$ & $\begin{array}{c}\text { Porcentaje de } \\
\text { población de } 15 \text { a } \\
24 \text { años }\end{array}$ \\
\hline Álvaro Obregón & 82 & $19.34 \%$ & 687,020 & $7.98 \%$ & 134,115 & $8.22 \%$ \\
\hline Coyoacán & 66 & $15.57 \%$ & 640,423 & $7.44 \%$ & 121,634 & $7.46 \%$ \\
\hline Benito Juárez & 60 & $14.15 \%$ & 360,478 & $4.19 \%$ & 56,718 & $3.48 \%$ \\
\hline Tlalpan & 50 & $11.79 \%$ & 581,781 & $6.76 \%$ & 117,415 & $7.20 \%$ \\
\hline Gustavo A. Madero & 30 & $7.08 \%$ & $1,235,542$ & $14.36 \%$ & 232,379 & $14.25 \%$ \\
\hline La Magdalena Contreras & 22 & $5.19 \%$ & 222,050 & $2.58 \%$ & 43,129 & $2.64 \%$ \\
\hline Cuauhtémoc & 21 & $4.95 \%$ & 516,255 & $6.00 \%$ & 90,941 & $5.58 \%$ \\
\hline \begin{tabular}{|l|} 
Iztapalapa \\
\end{tabular} & 21 & $4.95 \%$ & $1,773,343$ & $20.61 \%$ & 352,753 & $21.63 \%$ \\
\hline Miguel Hidalgo & 19 & $4.48 \%$ & 352,640 & $4.10 \%$ & 63,542 & $3.90 \%$ \\
\hline Venustiano Carranza & 14 & $3.30 \%$ & 462,806 & $5.38 \%$ & 82,942 & $5.09 \%$ \\
\hline Xochimilco & 14 & $3.30 \%$ & 369,787 & $4.30 \%$ & 73,683 & $4.52 \%$ \\
\hline Iztacalco & 11 & $2.59 \%$ & 411,321 & $4.78 \%$ & 74,652 & $4.58 \%$ \\
\hline Azcapotzalco & 10 & $2.36 \%$ & 441,008 & $5.12 \%$ & 80,198 & $4.92 \%$ \\
\hline Cuajimalpa & 3 & $0.71 \%$ & 151,222 & $1.76 \%$ & 29,748 & $1.82 \%$ \\
\hline Tláhuac & 1 & $0.24 \%$ & 302,790 & $3.52 \%$ & 57,957 & $3.55 \%$ \\
\hline Milpa Alta & 0 & $0.00 \%$ & 96,773 & $1.12 \%$ & 19,060 & $1.17 \%$ \\
\hline Total & 424 & $100.00 \%$ & $8,605,239$ & $100.00 \%$ & $1,630,866$ & $100.00 \%$ \\
\hline
\end{tabular}

En la Tabla 1 se aprecia que las cuatro delegaciones con mayor participación se encuentran cerca del ITAM: misma delegación, Álvaro Obregón, o delegación colindante, Coyoacán, Benito Juárez y Tlalpan. En el orden de participación hay dos casos que no concuerdan con la explicación de cercanía. Gustavo A. Madero, en el quinto lugar, que está lejos del ITAM y La Magdalena Contreras, en el sexto lugar, que está muy cerca. Esta variación podría explicarse por el porcentaje de la población total que habita en esas delegaciones: Gustavo A. Madero es la segunda delegación más poblada, mientras que en La Magdalena Contreras habita menos del 3\% de la población del Distrito Federal. Dado que Iztapalapa es la delegación con mayor población, su posición después de Gustavo A. Madero debe explicarse en función no sólo de la distancia y del número de habitantes. En la tabla 1 también se observa que no es necesario considerar sólo el rango de edades entre 15 y 24 años de la población en cada delegación ya que los porcentajes en este intervalo son similares a los porcentajes totales.

La Tabla 2 muestra la participación del ITAM en los alumnos que estudian Ingeniería o Tecnología en cada delegación. Estos resultados refuerzan la hipótesis de localidad, ya que el porcentaje de participación del ITAM es mayor en las delegaciones cercanas al Instituto, incluyendo a $\mathrm{La}$ Magdalena Contreras. 


\begin{tabular}{|l|l|r|r|r|r|}
\hline \multicolumn{1}{|c|}{ Delegación } & $\begin{array}{l}\text { Total de la } \\
\text { población de } \\
\text { 18 años y más } \\
\text { que estudia } \\
\text { ingeniería y } \\
\text { tecnología }\end{array}$ & $\begin{array}{c}\text { Número de } \\
\text { alumnos } \\
\text { inscritos }\end{array}$ & $\begin{array}{l}\text { Porcentaje de } \\
\text { Participación }\end{array}$ & $\begin{array}{l}\text { Porcentaje de de } \\
\text { alumnos } \\
\text { inscritos }\end{array}$ & $\begin{array}{l}\text { Porcentaje de la } \\
\text { población de 18 } \\
\text { años y más que } \\
\text { estudia ingeniería } \\
\text { y tecnología }\end{array}$ \\
\hline Álvaro Obregón & 19,678 & 82 & $0.42 \%$ & $19.34 \%$ & $7.21 \%$ \\
\hline Coyoacán & 31,325 & 66 & $0.21 \%$ & $15.57 \%$ & $11.47 \%$ \\
\hline Benito Juárez & 25,308 & 60 & $0.24 \%$ & $14.15 \%$ & $9.27 \%$ \\
\hline Tlalpan & 20,883 & 50 & $0.24 \%$ & $11.79 \%$ & $7.65 \%$ \\
\hline Gustavo A. Madero & 38,324 & 30 & $0.08 \%$ & $7.08 \%$ & $14.04 \%$ \\
\hline La Magdalena Contreras & 5,623 & 22 & $0.39 \%$ & $5.19 \%$ & $2.06 \%$ \\
\hline Cuauhtémoc & 18,741 & 21 & $0.11 \%$ & $4.95 \%$ & $6.86 \%$ \\
\hline Iztapalapa & 34,636 & 21 & $0.06 \%$ & $4.95 \%$ & $12.69 \%$ \\
\hline Miguel Hidalgo & 16,636 & 19 & $0.11 \%$ & $4.48 \%$ & $6.09 \%$ \\
\hline Venustiano Carranza & 13,209 & 14 & $0.11 \%$ & $3.30 \%$ & $4.84 \%$ \\
\hline Xochimilco & 8,936 & 14 & $0.16 \%$ & $3.30 \%$ & $3.27 \%$ \\
\hline Iztacalco & 12,360 & 11 & $0.09 \%$ & $2.59 \%$ & $4.53 \%$ \\
\hline Azcapotzalco & 17,318 & 10 & $0.06 \%$ & $2.36 \%$ & $6.34 \%$ \\
\hline Cuajimalpa & 4,222 & 3 & $0.07 \%$ & $0.71 \%$ & $1.55 \%$ \\
\hline Tláhuac & 4,796 & 1 & $0.02 \%$ & $0.24 \%$ & $1.76 \%$ \\
\hline Milpa Alta & 1,051 & 0 & $0.00 \%$ & $0.00 \%$ & $0.38 \%$ \\
\hline Total & 273,046 & 424 & $0.16 \%$ & $100 \%$ & $100.00 \%$ \\
\hline
\end{tabular}

Tabla 2. Participación del ITAM entre la población que estudia Ingeniería y Tecnología

Si se considera el nivel socioeconómico de sus habitantes, pueden ordenarse las delegaciones por el porcentaje de la población de mayores ingresos (A/B) que las habitan. La tabla 3 muestra este porcentaje. Se puede observar, por ejemplo, que el $18.43 \%$ de la población que pertenece al nivel más alto vive en la delegación Coyoacán, mientras que sólo el $0.02 \%$ habita en Milpa Alta. Las cinco delegaciones en las que vive el mayor porcentaje de la población $\mathrm{A} / \mathrm{B}$ concuerdan con las cinco delegaciones de las que hay más alumnos de Ingeniería en el ITAM, aunque en distinto orden.

\begin{tabular}{|c|c|c|c|c|c|c|}
\hline Delegación & $\begin{array}{c}\text { Población } \\
\text { total }\end{array}$ & $\begin{array}{c}\text { Población } \\
\text { A/B }\end{array}$ & $\begin{array}{c}\text { Población } \\
\text { C+ }\end{array}$ & $\begin{array}{c}\text { Porcentaje } \\
\text { de la } \\
\text { población } \\
\text { A/B }\end{array}$ & $\begin{array}{c}\text { Porcentaje } \\
\text { de la } \\
\text { población } \\
\text { C+ }\end{array}$ & $\begin{array}{c}\text { Porcentaje de } \\
\text { la población } \\
\text { A/B o C+ }\end{array}$ \\
\hline Coyoacán & 683,087 & 141,118 & 183,968 & $18.43 \%$ & $13.50 \%$ & $15.27 \%$ \\
\hline Benito Juárez & 360,962 & 119,725 & 150,890 & $15.63 \%$ & $11.07 \%$ & $12.71 \%$ \\
\hline Tlalpan & 637,685 & 82,185 & 121,373 & $10.73 \%$ & $8.91 \%$ & $9.56 \%$ \\
\hline Gustavo A. Madero & $1,279,245$ & 73,954 & 163,436 & $9.66 \%$ & $11.99 \%$ & $11.15 \%$ \\
\hline Álvaro Obregón & 727,684 & 72,710 & 108,997 & $9.49 \%$ & $8.00 \%$ & $8.54 \%$ \\
\hline Iztapalapa & $1,794,190$ & 54,174 & 162,740 & $7.07 \%$ & $11.94 \%$ & $10.19 \%$ \\
\hline Miguel Hidalgo & 346,190 & 51,770 & 82,037 & $6.76 \%$ & $6.02 \%$ & $6.29 \%$ \\
\hline Cuauhtémoc & 513,382 & 39,084 & 86,380 & $5.10 \%$ & $6.34 \%$ & $5.89 \%$ \\
\hline Azcapotzalco & 451,618 & 33,036 & 90,104 & $4.31 \%$ & $6.61 \%$ & $5.78 \%$ \\
\hline Xochimilco & 409,556 & 20,583 & 36,738 & $2.69 \%$ & $2.70 \%$ & $2.69 \%$ \\
\hline La Magdalena Contreras & 234,261 & 19,368 & 29,617 & $2.53 \%$ & $2.17 \%$ & $2.30 \%$ \\
\hline Cuajimalpa & 158,502 & 18,039 & 19,880 & $2.36 \%$ & $1.46 \%$ & $1.78 \%$ \\
\hline Venustiano Carranza & 486,735 & 17,102 & 51,443 & $2.23 \%$ & $3.77 \%$ & $3.22 \%$ \\
\hline Iztacalco & 418,040 & 14,123 & 42,048 & $1.84 \%$ & $3.09 \%$ & $2.64 \%$ \\
\hline Tláhuac & 318,252 & 8,677 & 29,703 & $1.13 \%$ & $2.18 \%$ & $1.80 \%$ \\
\hline Milpa Alta & 96,927 & 143 & 3,466 & $0.02 \%$ & $0.25 \%$ & $0.17 \%$ \\
\hline Total & $8,916,316$ & 765,791 & $1,362,820$ & $100.00 \%$ & $100.00 \%$ & $100.00 \%$ \\
\hline
\end{tabular}

Tabla 3. Porcentaje de población en los niveles socioeconómicos más altos 
Con el análisis de la información estadística y geográfica llevado a cabo en esta sección es posible conocer la influencia que tienen las variables de cercanía y nivel de ingresos los cuales son factores que influyen de manera considerable en la inscripción a alguno de los programas de Ingeniería del ITAM. Con el objetivo de dar un sustento formal a esta relación se calculó un modelo de regresión lineal múltiple para identificar estadísticamente la naturaleza y la fuerza de la relación entre la variable dependiente "número de alumnos inscritos" y las variables independientes "población con nivel socioeconómico tipo A/B y tipo C+ en la delegación" y "distancia entre el ITAM y la delegación".

La ecuación para el modelo planteado queda como se muestra a continuación:

$$
\mathrm{N}_{\mathrm{i}}=\mathrm{a}+\mathrm{b}_{1} * \mathrm{PABC}_{\mathrm{i}}+\mathrm{b}_{2} * \mathrm{D}_{\mathrm{i}}
$$

Donde:

$\mathrm{Ni}=$ Número de alumnos inscritos al ITAM provenientes de la delegación i.

PABCi $=$ Suma de la población con nivel socioeconómico tipo $\mathrm{A} / \mathrm{B}$ y $\mathrm{C}+$ de la delegación i.

$\mathrm{Di}=$ Distancia en kilómetros entre el ITAM y el centro de la delegación i.

Al estimar los parámetros de la regresión se obtuvieron los resultados mostrados en la Tabla 4. De acuerdo al valor del coeficiente de correlación múltiple, $r=0.83$, se puede decir que las variables PABCi ("Suma de la población con nivel socioeconómico tipo $\mathrm{AB}$ y $\mathrm{C}+$ de la delegación i”) y Di ("Distancia entre el ITAM y el centro de la delegación i") se encuentran asociadas fuertemente con la variable dependiente, "Número de alumnos inscritos provenientes de la delegación i". Por otro lado, de acuerdo al coeficiente de determinación, $r^{2}$, podemos decir que aproximadamente el $69 \%$ de las inscripciones pueden ser explicadas por el nivel socioeconómico y la distancia entre el ITAM y el centro de la delegación.

La ecuación de regresión múltiple estimada se muestra a continuación:

$$
\begin{gathered}
\mathrm{N}_{\mathrm{i}}=19.55+0.00018 * \mathrm{PABC}_{\mathrm{i}}+-1.14 * \mathrm{D}_{\mathrm{i}} \\
\text { Tabla 4. Resultados de la regresión múltiple }
\end{gathered}
$$

\begin{tabular}{|l|l|}
\hline Coeficiente de correlación múltiple, $r$ & 0.831 \\
\hline Observaciones & 16 \\
\hline
\end{tabular}

La granularidad del estudio, las 16 delegaciones políticas del Distrito Federal, puede ser mejorada si se analizan los datos a nivel de cada código 
postal. Esto aumenta la precisión de los datos al reducir el área geográfica a considerar y puede permitir estudiar con un poco de mayor detalle la influencia de la distancia para cada nivel socioeconómico. De la población total de alumnos fue posible determinar en 333 casos la distancia del ITAM al centro geográfico del código postal correspondiente y el nivel socioeconómico (promedio) de éste reportado en el Mapa Mercadológico de la Ciudad de México.

Los 333 casos se agruparon en 60 intervalos de acuerdo a la distancia (en $\mathrm{km}$ ) de cada código postal al ITAM. En las figuras 4 a 8 se grafica el número de alumnos inscritos que viven en cada uno de estos intervalos, correspondientes a 4 diferentes agrupaciones de niveles socioeconómicos. Es interesante notar que el 67\% del total de los alumnos vive a menos de $10 \mathrm{~km}$ de distancia del ITAM. Puede observarse en las figuras que la distribución espacial de los alumnos es diferente para cada una de las agrupaciones de niveles socioeconómicos consideradas. Por ejemplo, no hay alumnos inscritos correspondientes al grupo más privilegiado que vivan a más de $11 \mathrm{~km}$ del ITAM, mientras que varios de los alumnos de los niveles más bajos, y que gozan de ayuda financiera del Instituto, sí se desplazan una distancia mayor.

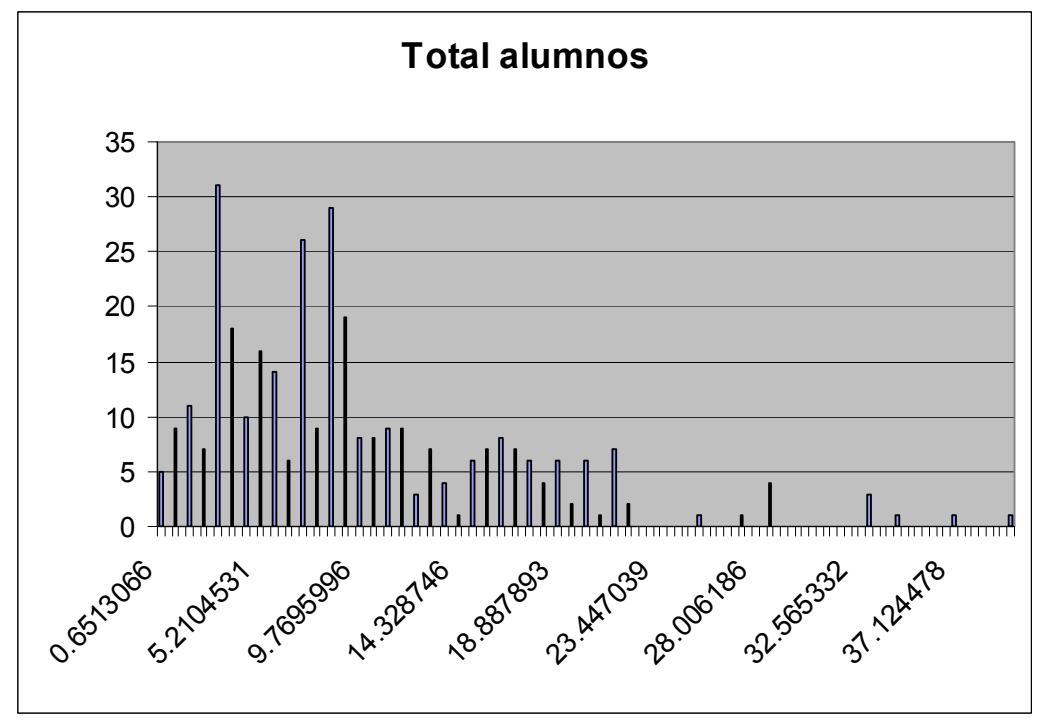

Figura 4. Distancia a la que viven los estudiantes actuales de Ingeniería 


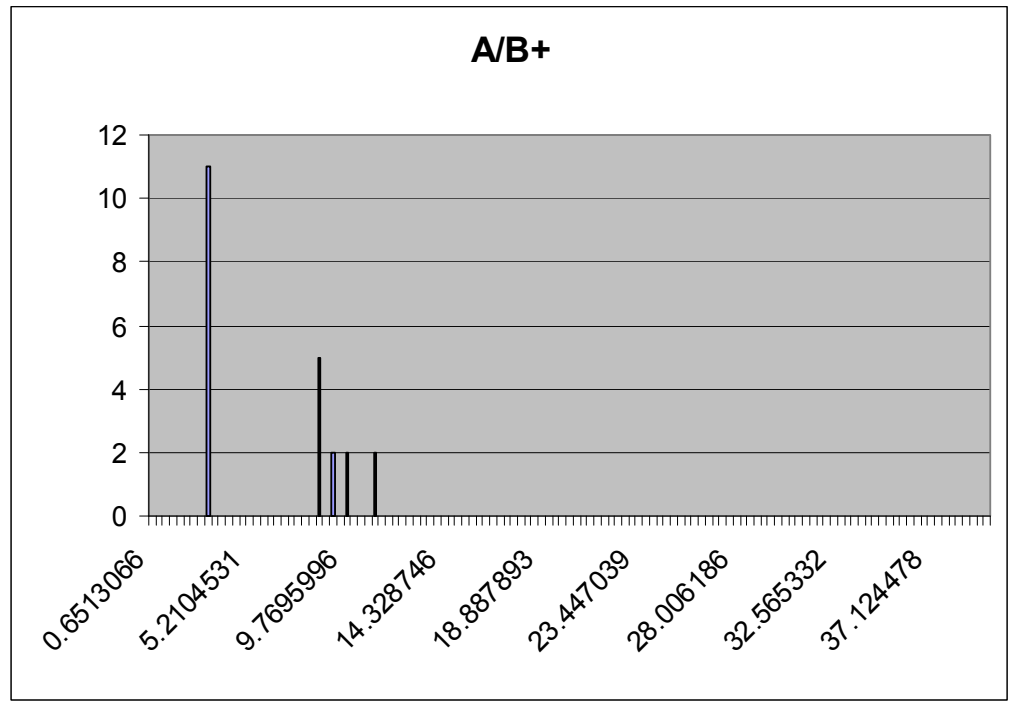

Figura 5. Distancia a la que viven los estudiantes de los niveles $A / B+$ $(6.61 \%$ del total)

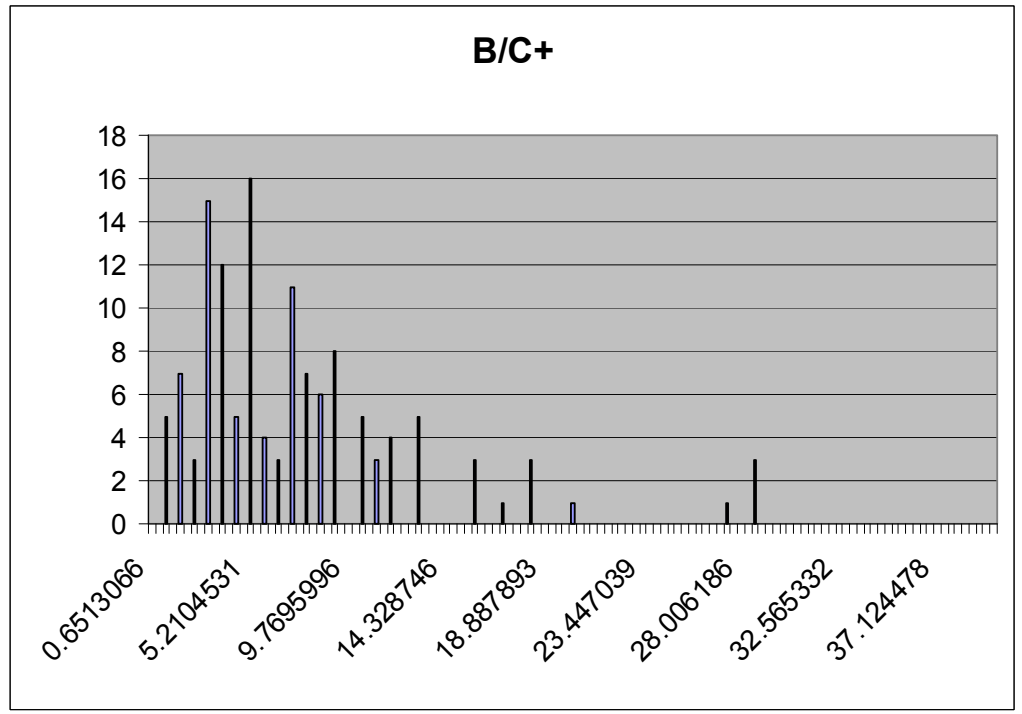

Figura 6. Distancia a la que viven los estudiantes de los niveles $\mathrm{B} / \mathrm{C}+$ (39.34\% del total) 


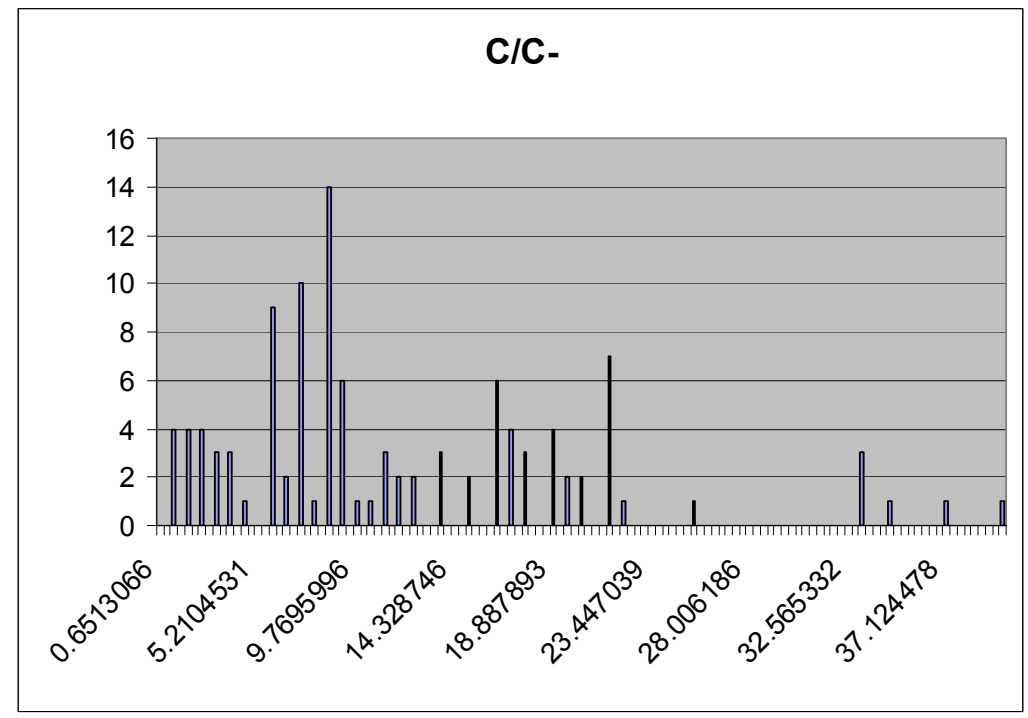

Figura 7. Distancia a la que viven los estudiantes de los niveles C/C(33.33\% del total)

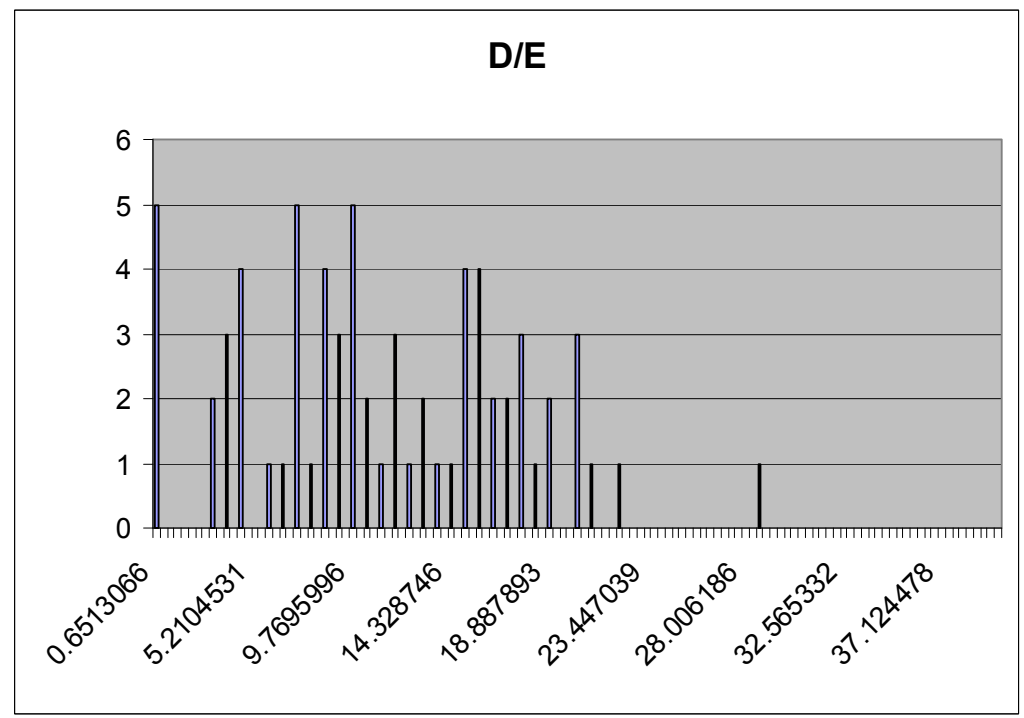

Figura 8. Distancia a la que viven los estudiantes de los niveles D/E (20.72\% del total)

\section{CONCLUSIONES}

Mediante el uso de herramientas computacionales sencillas se determinó que la distribución geográfica de los alumnos de Ingeniería del ITAM no 
es homogénea en la Ciudad México. Los resultados obtenidos por delegación y por rangos de distancia más pequeños sugieren que esta distribución puede explicarse, al menos parcialmente, por una hipótesis de localidad, que establece que las universidades atraen más alumnos de delegaciones cercanas a ellas, y por el nivel socioeconómico que se necesita para pagar las colegiaturas de una universidad privada. El prestigio público del ITAM y de sus programas (Reforma, 2006; El Universal, 2007), así como los programas de ayuda financiera que ofrece, pueden ser factores que expliquen el resto de la variabilidad en el número de alumnos inscritos.

La distribución espacial encontrada en el presente caso de estudio confirma los resultados publicados en investigaciones anteriores en Estados Unidos y su aplicabilidad en el contexto local de un área metropolitana grande de Latinoamérica. El artículo incorpora además el uso de los niveles socioeconómicos de los hogares de los alumnos y su relación con la distancia al ITAM y encuentra que los resultados de localidad se aplican sobretodo para alumnos que pertenecen a los niveles económicamente más privilegiados.

Los resultados obtenidos en este caso de estudio pueden ser utilizados por las autoridades administrativas del ITAM para apoyar o contrarrestar la influencia positiva o negativa de los factores estudiados. Para aumentar el número de alumnos que solicitan inscribirse al Instituto se podría decidir, por ejemplo, aumentar los esfuerzos de promoción en ciertas partes de la Ciudad de México, tener un servicio de autobús directo desde las zonas más alejadas de manera que el transporte se vuelva más sencillo y difundir de manera más agresiva el extenso programa de becas y préstamos con que cuenta el ITAM.

Aunque la escasez de datos demográficos precisos y la diversidad de agrupaciones con las que se reportan los datos en diferentes fuentes en México dificultan la realización de un análisis más exacto, el presente caso de estudio logró recabar datos muy interesantes sobre la distribución geográfica de los alumnos de Ingeniería del ITAM y sobre su nivel socioeconómico que pueden servir de base para un estudio más profundo. Los resultados obtenidos pueden ser ampliados incorporando al estudio el análisis de otros factores que podrían explicar aun más la variabilidad de la distribución geográfica no homogénea de los alumnos, como el prestigio del Instituto, la cantidad y la calidad de la información sobre los programas de ayuda financiera, la ubicación de universidades que son competencia para el ITAM o el medio y el tiempo de traslado hasta el Instituto. Otra área de oportunidad para continuar con la investigación en el futuro es incorporar datos longitudinales al estudio para determinar cómo varía a través del tiempo la relación de la distribución geográfica y el nivel socioeconómico de los estudiantes. 


\section{LECTURAS ADICIONALES RECOMENDADAS}

Miller, F. (2007). GIS Tutorial for Marketing, ESRI Press.

Pick, J. (2008). Geo-Business: GIS in the Digital Organization, Wiley.

Sherman, G. (2008). Desktop GIS: Mapping the Planet with Open Source Tools, Pragmatic Bookshelf.

\section{BIBLIOGRAFÍA}

ANUIES. (2004). Anuario estadístico - Población Escolar de Licenciatura y Técnico Superior en Universidades e Institutos Tecnológicos. México: Asociación Nacional de Universidades e Instituciones de Educación Superior.

Barrera, A. (2000). Proyecto de Impulso Estratégico para la Ingeniería en Computación del ITAM. Tesis de la Maestría en Tecnologías de Información y Administración, ITAM, Ciudad de México, México.

Buró de Investigaciones de Mercado. (2004). Mapa Mercadológico de la Ciudad de México, México.

Christie, R. M., and Ferris, M. (2004). Spatial Analysis for Enrollment Planning in Higher Education. Proceedings of the 24th Annual ESRI International User Conference. Recuperado el 27 de febrero de 2007, de http:/gis2.esri.com/library/ userconf/educ04/papers/pap5072.pdf

El Universal (2007, 16 de abril). Mejores Universidades 07 - Guía de Universidades y Programas de Licenciatura. El Universal, suplemento especial.

George, J. F., Valacich, J. S., \& Valor, J. (2005). Does Information Systems Still Matter? Lessons for a Maturing Discipline. Communications of the Association for Information Systems, 16, 219232.

Granger, M., Dick, G., Luftman, J., Van Slyke, C., \& Watson, R. (2007a). Information Systems Enrollments: Can They be Increased? Communications of the Association for Information Systems, 20, 649659.

Granger, M., Dick, G., Jacobson, C., \& Van Slyke, C. (2007b). Information Systems Enrollments: Challenges and Strategies. Journal of Information Systems Education, 18(3), 303-311.

Greene, R. P., \& Pick, J. B. (2006). Exploring the Urban Community: A GIS Approach. Upper Saddle River, NJ: Prentice Hall.

Herries, J., and Marble, D. F. (1997). A Model for the Use of GIS Technology in College and University Admissions Planning. Proceedings of the 17th Annual ESRI International User Conference, Recuperado el 27 de febrero de 2007, de http://gis.esri.com/library/userconf/proc97/proc97/to250/pap218/p218 .htm.

INEGI. (2007). Sistema Municipal de Base de Datos. México: Instituto Nacional de Geografía, Estadística e Informática. Consultado el 3 de febrero 2007, de http://sc.inegi.gob.mx/simbad/. 
Looney, C., \& Akbulut, A. (2007). Combating the IS enrollment crisis: The Role of Effective Teachers in Introductory IS Courses. Communications of the Association for Information Systems, 19, 781805.

Marble, D. F., Mora, V. J., and Herries, J. P. (1995). Applying GIS Technology to the Freshman Admissions Process at a Large University. Proceedings of the 15th Annual ESRI International User Conference. Recuperado el 27 de febrero de 2007, de http://gis.esri.com/library/userconf/proc95/to200/p182.html.

Marble, D. F., Mora, V. J., and Granados, M. (1997). Applying GIS Technology and Geodemographics to College and University Admissions Planning: Some Results from the Ohio State University. Proceedings of the 17th Annual ESRI International User Conference. Recuperado el 27 de febrero de 2007, de http://gis.esri.com/library/userconf/proc97/proc97/to550/pap501/p501 .htm.

Martin, R. N. (2000). The Shrinking Student Pool and Higher Education: An Example from Pennsylvania Proceedings of the 20th Annual ESRI International User Conference. Recuperado el 27 de febrero de 2007, de

http://gis.esri.com/library/userconf/proc00/professional/papers/PAP32 2/p322.htm.

Martin, R. N. (2001). Identifying Target Enrollment Areas to Improve Diversity. Proceedings of the 21st Annual ESRI International User Conference. Recuperado el 27 de febrero de 2007, de http://gis.esri.com/library/ userconf/proc01/professional/papers/pap445/p445.htm.

Martin, R. N. (2002). Identifying Target Enrollment Areas to Improve Diversity, Part 2. Proceedings of the 22nd Annual ESRI International User Conference. Recuperado el 27 de febrero de 2007, de http://gis.esri.com/library/ userconf/proc02/pap0618/p0618.htm.

Pick, J. B. (2005). Geographic Information Systems in Business. Hershey, PA: Idea Group Publishing.

Price, A. (2004). GIS and Undergraduate Engineering Recruitment: An Exploratory Study at DeVry University, Pomona. ASEE Annual Conference and Exposition, Salt Lake City, Utah.

Reforma (2006, 20 de agosto). Las Mejores Universidades 2006-2007. Reforma, suplemento mensual Universitarios.

Rushton, G., Armstrong, M. P., \& Lolonis, P. (1995). Small Area Student Enrollment Projections Based on a Modifiable Spatial Filter. SocioEconomic Planning Sciences, 29(3), 169-185,

Vegso, J. (2008). Enrollments and Degree Production at US CS Departments Drop Further in 2006-07. Computing Research News Online, 20(2). Recuperado el 21 de marzo de 2008, de http://www.cra.org/CRN/online.html. 
Zhou, Y., and Wu, J. (2005). Modeling University Enrollments with ArcGIS. Proceedings of the 25th Annual ESRI International User Conference. Recuperado el 27 de febrero de 2007, de http://gis2.esri.com/library/ userconf/educ05/papers/pap1262.pdf.

Nota del Editor: Las referencias anteriores contienen direcciones de WWW. (a) Dichas direcciones existían a la fecha de publicación pero no hay garantía alguna de que persistan en el tiempo, (b) su contenido puede cambiar en el tiempo y las siguientes versiones pueden no contener la información a la que se hizo referencia, (c) el autor o autores de dichas páginas (no AIS ni RELCASI) son los únicos responsables de su contenido, y (d) el autor de este articulo (no AIS ni RELCASI) es el único responsable de la exactitud de dicha dirección.

Marcelo Mejía Olvera se graduó de la licenciatura en Ingeniería Biomédica en la Universidad Autónomo Metropolitana (UAM) en 1982 y ha realizado estudios de Maestría en Ciencias de la Computación en la UAM, en Ingeniería Eléctrica en la División de Estudios de Posgrado de la Facultad de Ingeniería de la Universidad Nacional Autónoma de México, y en Redes Informáticas en la Escuela Superior de Electricidad en Francia. En 1989 obtuvo el grado de Doctor en Informática de la Universidad de Rennes I, en Francia. Desde 1989 labora como profesor de Tiempo Completo en el ITAM donde actualmente se desempeña como Director General de la División Académica de Ingeniería. Ha sido Vicepresidente de la Sociedad Mexicana de Ciencia de la Computación y Presidente del Consejo Nacional de Acreditación en Informática y Computación.

Quauhtli Martínez Zazueta se graduó como Ingeniero en Computación en 2004 en el ITAM y obtuvo el grado de Maestro en Tecnologías de Información y Administración, también en el ITAM, y el Mastère Spécialisé Manager Télécom, en el Instituto Nacional de Telecomunicaciones (INT) en Francia, en el año 2006. Desde el 2004 se ha desempeñado como Arquitecto de Tecnologías de Información en empresas establecidas en México como ING, AXA, Grupo Nacional Provincial, y a nivel internacional en Pacífico Seguros en Lima, Perú, donde labora actualmente. Su especialidad es la evaluación, diseño y construcción de Arquitecturas Orientadas a Servicios (SOA). 


\section{Política Editorial}

RELCASI está principalmente dirigida a investigadores de habla hispana en el área de Administración de Sistemas de Información (MIS por sus siglas en Inglés). Los artículos son académicamente rigurosos sin sacrificar la claridad, estilo, simplicidad y contribución práctica que los hace atractivos a profesionales de la disciplina. En consecuencia, la audiencia de esta revista está compuesta no sólo por investigadores de MIS, sino también por profesionales y administradores en el área de tecnologías de información.

Todos los artículos son escritos, revisados y publicados en español; sin embargo, estos contendrán título, resumen y palabras claves en español y en inglés.

RELCASI es una publicación arbitrada que se presenta en formato impreso y en línea. La versión impresa de RELCASI está disponible a pedido (y próximamente bajo subscripción). La versión en línea se provee a través de la Asociación de Sistemas de Información. El proceso de evaluación se realizará con al menos dos examinadores. La identidad del editor asociado y de los examinadores no será del conocimiento del autor, y ni los examinadores ni el editor asociado conocerán la identidad del autor. Una ronda del proceso de evaluación tomará alrededor de 90 días.

La revista incluye principalmente artículos de investigación desarrollados con un marco teórico robusto y que incluyan una adecuada revisión de literatura. Los artículos podrán ser de investigación empírica (cualitativa o cuantitativa), conceptuales, encuestas de corrientes de investigación, o encuestas de la industria de TI en países en desarrollo. Los artículos de investigación empírica, deben proveer una amplia justificación y descripción de la colección de datos, metodología y técnicas analíticas. Estudios de caso, artículos pedagógicos, revisión de libros, y debates y ensayos de opinión serán considerados pero no formarán el grueso de la publicación. Artículos con un alto contenido técnico y bajo contenido gerencial/administrativo no son recomendados y sólo serán aceptados cuando sean altamente relevantes o innovadores. Los artículos deberán tener una extensión no mayor a las 8.000 palabras. 


\section{Editorial Policy}

RELCASI is primarily directed to Spanish speaking researchers in the area of Management Information Systems (MIS). Articles will have academic rigor without sacrificing clarity, style, simplicity, and a practical contribution that will also make them attractive to practitioners. Therefore, its audience includes both academics and practitioners of MIS and IT.

Articles are written, reviewed, and published in Spanish; however, their title, abstract, and keywords will also be published in English.

RELCASI is a double-blind peer-reviewed journal that is both in-print and on-line. The print version is currently provided on-demand and we will soon have a subscription service. The on-line version is available through the Association for Information Systems. The double-blind peer-review process will involve an associate editor and a minimum of two academic peers. We aim to have a round of the review process take no more than 90 days.

The journal will primarily comprise of research articles developed with a robust theoretical framework that include an appropriate literature review. The articles could be qualitative or quantitative, conceptual, research stream surveys, or surveys that relate to IT/MIS in developing countries. Empirical research articles must include a clear, comprehensive, and concise description of the methodology, data collection, and analytical techniques used. Case studies, pedagogical articles, book reviews, debates, and opinion papers will be considered but will not make the bulk of the journal. Articles with a high technical and low managerial content are not encouraged but may be accepted if highly relevant or innovative. Articles may not include more than 8,000 words. 


\section{Solicitud de Artículos \\ Call for Articles}

RELCASI está permanentemente en la búsqueda de artículos en español en el área de sistemas de información (MIS), la cual incluye tópicos relacionados a la adopción, administración, uso, e impactos de la tecnología de información (TI). Tópicos populares incluyen (pero no están limitados a) los siguientes:

- Estudios inter-culturales (dentro de países latinoamericanos o comparaciones con países desarrollados) que comparen antecedentes e impactos de la TI en organizaciones

- Modelos de bases de datos y estructuras de sistemas de información a nivel empresarial o global

- Factores culturales que influyen en el desarrollo efectivo de sistemas de información a gran escala

- Costos y Retornos de Inversión esperados en la implementación de tecnologías de información

- Impacto de TI emergente en pequeñas y medianas empresas (PYMEs)

- El rol de TI en mejorar la ventaja competitiva de las PYMEs

- Infraestructura de sistemas de información

- Recursos humanos en sistemas de información

- Impacto de la TI en la productividad individual

- Programas personalizados vs encapsulados

- Efectos de obligar el uso de TI específicas en subsidiarias locales

- Tercerización (“outsourcing”) / Offshoring / Nearshoring

- Debates acerca de implementaciones globales

- Procura de TI en países en vías de desarrollo

- Uso, difusión y legislación de programas de código abierto en Latinoamérica

- Costo total: programas, adaptación, consultoría y entrenamiento

- Como programas de código abierto pueden contribuir al desarrollo

- Uso, venta, e implementación de paquetes globales en economías locales

- Impacto de estilos gerenciales en el uso y desarrollo de TI

- Ejecución de contratos de TI en una economía global

- La paradoja de la productividad de la TI en Latinoamérica

- Implementación y adaptación de paquetes de programas

- Implementaciones globales

- Transferencia de tecnología

- Aspectos económicos y financieros de la compra, desarrollo e implementación de TI 
- Debates en sistemas de entrenamiento de TI (para expertos y usuarios)

- El valor del negocio de la TI

- Soporte local vs soporte a larga distancia

Los artículos pueden utilizar cualquiera de las siguientes modalidades de acuerdo al contexto y metodología.

1. Investigación empírica

a. Cualitativa (perspectivas positivistas o interpretativas): desarrollo o comprobación de teorías: estudios de caso, estudios de casos múltiples, investigación-acción

b. Cuantitativa: comprobación de teorías: experimentos, encuestas, estudios de caso, archivos

2. Encuestas de corrientes de investigación: revisión de literatura usando narrativa o meta-análisis

3. Encuestas de la industria de TI en países en desarrollo. Debido a la falta de difusión del conocimiento de TI en países en desarrollo es importante publicar artículos que provean una visión general de la situación de la industria de TI en estos países. Los artículos pueden ser reportes académicos que provean una clara representación de la industria de TI y/o su relación con otras industrias y el gobierno.

4. Conceptual: desarrollo de nuevas teorías/modelos desde literatura existente, observación de hechos y argumentos lógicos

5. Diseño de la investigación: desarrollo de artefactos para resolver problemas relevantes que profesionales de la TI enfrentan en países en desarrollo. Algunos ejemplos pueden incluir: desarrollo de herramientas y aplicaciones innovadoras de TI, nuevos métodos para gerenciar TI en países en desarrollo, etc.

Artículos con un alto contenido técnico y bajo contenido gerencial/administrativo no son recomendados y solo serán aceptados cuando sean altamente relevantes o innovadores. La revista incluirá principalmente artículos teóricos y de investigación que han sido desarrollados en un marco teorético robusto, incluyen una adecuada revisión de literatura y proveen una amplia justificación y descripción de la metodología y técnicas analíticas. Estudios de caso, artículos pedagógicos, revisión de libros, y debates y ensayos de opinión serán considerados pero no formarán parte del grueso de la publicación. 


\section{AUDIENCIA}

La revista está principalmente dirigida a investigadores de MIS de habla hispana. Los artículos serán académicamente rigurosos sin sacrificar la claridad, estilo y simplicidad que hace que estos artículos sean atractivos a profesionales de la disciplina En consecuencia, la revista será atractiva no solo para investigadores de MIS y sino también para profesionales.

\section{IDIOMA}

Todos los artículos serán escritos, revisados y publicados en español; sin embargo, el título, palabras claves y resumen deberán ser incluidos en inglés y español

\section{FORMATO}

La revista es una publicación arbitrada que se presentará en formato impreso y en línea. La versión impresa de la revista estará disponible a pedido. La versión electrónica será publicada en el sitio de AIS. Los artículos deberán tener una extensión no mayor a las 8.000 palabras.

El proceso de evaluación se realizara con al menos dos árbitros. La identidad del editor asociado y de los examinadores no será del conocimiento del autor y estos tampoco conocerán la identidad del autor. Una ronda del proceso de evaluación deberá tomar alrededor de 90 días.

Los artículos deben ser enviados directamente a través del sistema automatizado en http://mc.manuscriptcentral.com/relcasi Para preguntas y sugerencias envíe un correo electrónico a editor@relcasi.org Información adicional está disponible en www.relcasi.org 